\title{
Sensitivity enhancement in off-axis integrated cavity output spectroscopy
}

\author{
R. Centeno, " J. Mandon, S. M. Cristescu, and F. J. M. Harren \\ Life Science Trace Gas Facility, Radboud University, P.O. Box 9010, NL-6500 GL, The Netherlands \\ *r.centeno@science.ru.nl
}

\begin{abstract}
We report on a detailed model of an improved three mirror offaxis integrated cavity output spectroscopy (OA-ICOS) setup, which reinjects the light reflected by the optical cavity. The model simulates the impact of design parameters on instrument sensitivity and can be used for any off-axis configuration. We demonstrate the application of this model for the real-time detection of ethylene with a pulsed quantum cascade laser (QCL). The three mirror OA-ICOS scheme provides a 10 times increase in signal-to-noise ratio as compared to standard OA-ICOS, resulting in a noise equivalent absorption sensitivity of $1.5 \times 10^{-8} \mathrm{~cm}^{-1} \mathrm{~Hz}^{-1 / 2}$.

(C)2014 Optical Society of America

OCIS codes: (300.6340) Spectroscopy, infrared; (300.6360) Spectroscopy, laser; (120.6200) Spectrometers and spectroscopic instrumentation; (140.5965) Semiconductor lasers, quantum cascade; (120.4570) Optical design of instruments.
\end{abstract}

\section{References and links}

1. A. O'Keefe, J. J. Scherer, and J. B. Paul, “CW integrated cavity output spectroscopy," Chem. Phys. Lett. 307(56), 343-349 (1999).

2. R. Engeln, G. Berden, R. Peeters, and G. Meijer, "Cavity enhanced absorption and cavity enhanced magnetic rotation spectroscopy," Rev. Sci. Instrum. 69(11), 3763-3769 (1998).

3. D. S. Baer, J. B. Paul, M. Gupta, and A. O'Keefe, "Sensitive absorption measurements in the near-infrared region using off-axis integrated-cavity-output spectroscopy," Appl. Phys. B 75(2-3), 261-265 (2002).

4. Y. A. Bakhirkin, A. A. Kosterev, C. Roller, R. F. Curl, and F. K. Tittel, "Mid-infrared quantum cascade laser based off-axis integrated cavity output spectroscopy for biogenic nitric oxide detection," Appl. Opt. 43(11), 2257-2266 (2004).

5. G. S. Engel, W. S. Drisdell, F. N. Keutsch, E. J. Moyer, and J. G. Anderson, "Ultrasensitive near-infrared integrated cavity output spectroscopy technique for detection of $\mathrm{CO}$ at 1.57 microm: new sensitivity limits for absorption measurements in passive optical cavities," Appl. Opt. 45(36), 9221-9229 (2006).

6. D. Herriott, H. Kogelnik, and R. Kompfner, "Off-axis paths in spherical mirror interferometers," Appl. Opt. 3(4), 523-526 (1964).

7. J. B. Paul, L. Lapson, and J. G. Anderson, "Ultrasensitive absorption spectroscopy with a high-finesse optical cavity and off-axis alignment," Appl. Opt. 40(27), 4904-4910 (2001).

8. E. Moyer, D. Sayres, G. Engel, J. S. Clair, F. Keutsch, N. Allen, J. Kroll, and J. Anderson, "Design considerations in high-sensitivity off-axis integrated cavity output spectroscopy," Appl. Phys. B 92, 467-474 (2008).

9. Y. A. Bakhirkin, A. Kosterev, R. Curl, F. Tittel, D. Yarekha, L. Hvozdara, M. Giovannini, and J. Faist, "Subppbv nitric oxide concentration measurements using $\mathrm{cw}$ thermoelectrically cooled quantum cascade laser-based integrated cavity output spectroscopy," Appl. Phys. B 82(1), 149-154 (2006).

10. P. Sturm and A. Knohl, "Water vapor $\delta^{2} \mathrm{H}$ and $\delta{ }^{18} \mathrm{O}$ measurements using off-axis integrated cavity output spectroscopy," Atmospheric Measurement Techniques 3(1), 67-77 (2010)

11. D. Marchenko, J. Mandon, S. Cristescu, P. Merkus, and F. Harren, "Quantum cascade laser-based sensor for detection of exhaled and biogenic nitric oxide," Appl. Phys. B 111(3), 359-365 (2013).

12. M. Silva, D. Sonnenfroh, D. Rosen, M. Allen, and A. O'Keefe, "Integrated cavity output spectroscopy measurements of NO levels in breath with a pulsed room-temperature QCL," Appl. Phys. B 81(5), 705-710 (2005).

13. R. Centeno, J. Mandon, S. M. Cristescu, and F. J. M. Harren, "Three mirror off axis integrated cavity output spectroscopy for the detection of ethylene using a quantum cascade laser," Sens. Actuators B Chem. 203, $311-$ 319 (2014).

14. D. S. Sayres, E. J. Moyer, T. F. Hanisco, J. M. St Clair, F. N. Keutsch, A. O’Brien, N. T. Allen, L. Lapson, J. N. Demusz, M. Rivero, T. Martin, M. Greenberg, C. Tuozzolo, G. S. Engel, J. H. Kroll, J. B. Paul, and J. G. Anderson, "A new cavity based absorption instrument for detection of water isotopologues in the upper troposphere and lower stratosphere," Rev. Sci. Instrum. 80(4), 044102 (2009).

15. E. Hecht, “Optics, 4th,” International edition, Addison-Wesley, San Francisco (2002). 
16. D. W. Allan, "Statistics of atomic frequency standards," Proc. IEEE 54(2), 221-230 (1966).

17. C. Dyroff, "Optimum signal-to-noise ratio in off-axis integrated cavity output spectroscopy," Opt. Lett. 36(7), $1110-1112(2011)$.

\section{Introduction}

In cavity-enhanced techniques the absorption path length can be increased up to several kilometers $[1,2]$, hence enhancing the sensitivity significantly. The development of off-axis integrated cavity output spectroscopy (OA-ICOS) [3-7] offered mechanical robustness and relatively simple alignment for field measurements [8], being used in numerous studies during the recent years, such as isotopic studies $[9,10]$ and breath analysis $[4,11,12]$. The method can straightforward be implemented into practical trace-gas detection schemes, offering great versatility and selectivity, making it less technically demanding and more cost effective than other laser-based methods. A major drawback of OA-ICOS is the significantly reduced cavity transmitted power due to highly reflective mirrors, which impact on the sensitivity of the sensor. It becomes critical when low power lasers and room-temperature detectors with limited detectivity are the only suitable or available devices for the designed system.

To overcome this drawback, we reported previously on an improved three mirror OAICOS configuration which recycles the light into the cavity [13]. The setup consisted of a pulsed distributed feedback QCL at room temperature in combination with an OA-ICOS cell for the detection of ethylene. Light emitted from the QCL was coupled into a high finesse cavity and the spectroscopic information was extracted from a measurement of the time integrated light intensity leaking out of the cavity. Here we present a detailed model on how the optimization process can be performed to achieve optical enhancement. The influence of different re-injection parameters and their effect on the signal-to-noise ratio (SNR) are presented and compared to the experimental results of real-time detection of ethylene. Practical considerations about the use of this simulation tool for other off-axis configurations are also discussed.

\section{Material and methods}

\section{$2.1 O A-I C O S$}

The OA-ICOS technique has been extensively reviewed in the literature [3-8]; here we briefly highlight the most relevant aspects. It is a laser-based spectroscopic method developed from cavity ring down spectroscopy. The laser light is injected into a closed optical cavity of length $\mathrm{L}$ formed by a pair of high-reflectivity mirrors with reflectivity $\mathrm{r}$. The laser beam is tuned over the molecular gas absorption feature of interest. The absorption strength of the gas inside the cavity is extracted from the time-integrated light intensity that leaks out at the rear mirror of the cavity onto the detector. In on-axis configuration, the light is only transmitted at specific wavelengths determined by the Free Spectral Range (FSR) of the cavity. If the laser light is injected off-axis into a cavity, a high number of cavity modes is excited, and the reflection spots on the mirrors either form a circle (spherical mirrors) or a Lissajous pattern (astigmatic mirrors) [14]. This reduces the FSR of the cavity proportional to the number of excited cavity modes. The angle between two adjacent spots on the mirror surface is determined solely by the geometry of the cavity. Based on the properties of the cavity, the reentrant condition can be fulfilled after only a few passes or reach extremely high values. This will result in a dense spectral mode structure of the optical cavity reducing or even cancelling strong power fluctuations as the on-axis configuration [5]. As such, OA-ICOS takes advantage of long path absorption spectroscopy without active locking to a single longitudinal cavity mode.

An additional mirror with a small entrance hole placed in front of the optical cavity reinjects the light reflected from the input mirror of the cavity. This is particularly desirable for a broad laser linewidth or an absorption cell with small diameter. In the first case, only a small portion of the light can be coupled inside a cavity mode, while in the latter configuration the cell can only slightly be aligned off-axis, thereby reducing the coupling to the cavity resonances and the optical noise only minimally. The absorption signal and the performance of the system can be significantly enhanced depending on various parameters of the re- 
injection mirror, most importantly the entrance position, the angle and its distance to the cavity. In addition, the optical power transmission of the complete system is improved, thereby counteracting the low detectivity of the detector.

\subsection{Re-injection model}

To simulate the effect of the re-injection mirror on the transmission power of the system we used a ray trace approach through all three mirrors with identical properties, varying the initial position $(\mathrm{x}, \mathrm{y})$, the incoming angle $\left(\alpha_{\mathrm{x}}, \alpha_{\mathrm{y}}\right)$ and the distance $\mathrm{d}$ between the re-injection mirror and the first cavity mirror. The model used in [13] was enhanced by means of a thick lens approach and corrected the matrices for astigmatism of the mirrors [15]. The incoming laser beam was modeled by a ring of smaller beams which cover its cross-sectional surface with a waist $\omega_{0}$ in the center of the cavity. We used the following notation to model the ray trace: $\mathrm{s}$ was the vector containing the position and angle of the set of beams in $\mathrm{x}$ - and $\mathrm{y}$-direction, $\mathrm{T}(\mathrm{d})$ translated the beam over a distance $\mathrm{d}, \mathrm{T}\left(\mathrm{R}_{\mathrm{i}}\right)$ reflected the beam off a mirror $\mathrm{i}$ with radius of curvature $R$ and $T(n)$ acted as a refraction surface with curvature $R$ and refractive index $n$. The relations between the different parameters are shown in Fig. 1(a), while the steps of the calculations for this model are visualized in Fig. 1(b). Starting at the re-injection mirror with coordinates $\left(\mathrm{x}, \alpha_{\mathrm{x}}, \mathrm{y}, \alpha_{\mathrm{y}}\right)$, we first translated the beam towards the cavity entrance mirror by

$$
s_{1}=T(d) \cdot s_{0}
$$

The beam then refracted on the front surface of the entrance mirror, propagated through its thickness $t$ and refracted again, according to

$$
s_{2}=T(n) \cdot T(t) \cdot T(n) \cdot s_{1}
$$

For each beam which entered the cavity the ray trace recursively calculated its path through the cavity until it either fulfilled the reentrant condition or hit the walls of the cavity. A cavity round trip was calculated using

$$
s_{3}=T\left(R_{2}\right) \cdot T(L) \cdot T\left(R_{3}\right) \cdot T(L) \cdot s_{2}
$$

where $L$ is the cavity length and $R_{2}$ and $R_{3}$ are the radii of curvature of the first and second cavity mirror, respectively. The number of round trips $\mathrm{m}$ inside of the cavity was calculated according to $\mathrm{m} 2 \theta=\mathrm{q} 2 \pi$, where $\mathrm{q}$ is an integer, $\mathrm{m}$ the number of round trips inside of the cavity and $2 \theta$ the angle between two adjacent spots on the mirror surface. The subsequent reflections and refractions occurring after the first cavity transmission were calculated by

$$
s_{4}=T\left(R_{1}\right) \cdot T(d) \cdot T(n) \cdot T(t) \cdot T\left(-R_{2}\right) \cdot T(t) \cdot T(n) \cdot s_{1}
$$

with $R_{1}$ the radius of curvature of the re-injection mirror. The minus sign in $T\left(-R_{2}\right)$ occurs due to the fact that the beam reflects off the back side of a concave mirror. To determine how many re-injections, n, occurred between these two mirrors, this calculation ran recursively until the beam either exited through the entrance hole or did not enter the cavity. The corresponding calculation of the enhancement ratio in power as a function of round trips has been previously described [13]. Note that only the power enhancement was calculated, as the optical noise may increase due to increased laser noise or etalon effects.

\#220903 - \$15.00 USD Received 12 Aug 2014; revised 23 Sep 2014; accepted 30 Sep 2014; published 4 Nov 2014

(C) 2014 OSA 17 November 2014 | Vol. 22, No. 23 | DOI:10.1364/OE.22.027985 | OPTICS EXPRESS 27987 

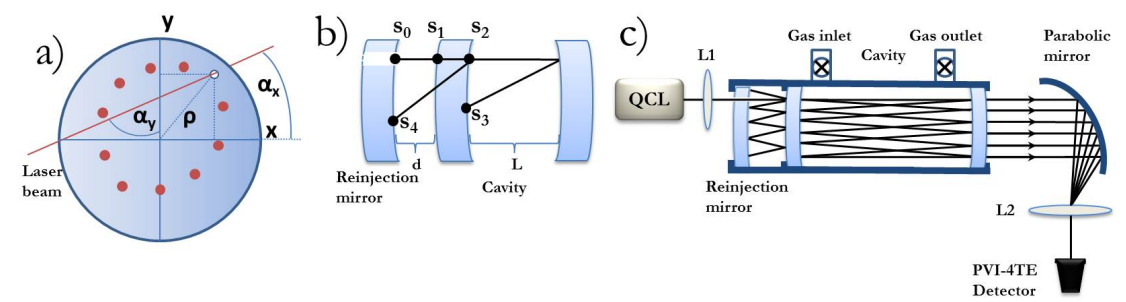

Fig. 1. Panel a: Geometric relations for the re-injection model. $\alpha_{\mathrm{y}}$ and $\alpha_{\mathrm{x}}$ represent the angles of the laser beam with the $\mathrm{y}$ - and $\mathrm{x}$-axis, respectively. Red dots indicate a typical elliptical spot pattern on the mirror. Panel b: Schematic of the steps during the ray trace calculations. Panel c: Experimental setup. QCL: quantum cascade laser; L1, L2: focusing and collimating lens, respectively; PVI-4TE detector: four stages thermoelectrically cooled detector.

\subsection{Experimental setup}

The experimental setup is shown in Fig. 1(c) and has been described in detail elsewhere [13]. A pulsed QCL with a center frequency of $965 \mathrm{~cm}^{-1}$ (Alpes Laser) at room temperature was used for ethylene detection. The laser emitted $30 \mathrm{~ns}$ pulses with a repetition rate of $1 \mathrm{MHz}$ with an average power of $10 \mathrm{~mW}$. To scan over the ethylene absorption line we applied a bias current ramp of $7 \mathrm{kHz}$ to the laser at sub-threshold level while maintaining the pulse height. With the help of a focusing lens $(\mathrm{ZnSe}$, diameter $25.4 \mathrm{~mm}, \mathrm{f}=30 \mathrm{~cm})$ the laser light was injected into the high finesse cavity formed by two spherical mirrors (ZnSe, diameter 50.8 $\mathrm{mm}, \mathrm{R}=50 \mathrm{~cm}$ ) with a reflectivity of $\mathrm{r}=99.6 \%$ at $10.3 \mu \mathrm{m}$ (II-VI Infrared) and a refractive index of $\mathrm{n}=2.4$. The cavity consisted of a vacuum-tight aluminum tube of $30 \mathrm{~cm}$ length (cell volume 0.61 , free spectral range of $500 \mathrm{MHz}$ ) mounted on two micrometer XY-translation stages for precise alignment. The mechanical movement of the translation stages was converted into an angular shift of the cavity, which was measured using a digital spirit level. The third mirror was mounted parallel to the cavity and on its axis. A parabolic mirror behind the cell $(\mathrm{f}=10 \mathrm{~cm})$ collected the light and a lens $(\mathrm{ZnSe}, \mathrm{f}=5 \mathrm{~cm})$ focused it onto the thermoelectrically cooled detector (VIGO PVI-4TE-10.6, $100 \mathrm{MHz}$ bandwidth, D* $=7.3 \cdot 10^{9}$ $\mathrm{cm} \mathrm{Hz}^{1 / 2} \mathrm{~W}^{-1}$ ). The signal from the detector was amplified by $40 \mathrm{~dB}$ (Femto DLPVA-100BLN-S) and low-pass filtered at $100 \mathrm{kHz}$. All data were sampled on a digital acquisition card (NI PCI-6259) and analyzed with a Lab VIEW program.

\section{Results}

The model considers only entrance points on the re-injection mirror such that $\mathrm{x}=\mathrm{y}$ starting from $\rho=0$ to the maximum of $\rho=24 \mathrm{~mm}$ with $\rho^{2}=x^{2}+y^{2}$ for constant $R, L, \omega_{0}$ and $r$ for all mirrors. Due to symmetry, only entrance points in the positive quarter of the entrance mirror needed to be considered. A measured beam waist of $\omega_{0}=0.21 \mathrm{~mm}$ at the center of the cavity was used as input for the model. We simulated the angular distribution of the transmission power as a function of off-axis radius $\rho$ and the ratio $\mathrm{d} / \mathrm{R}$ for an initial laser power of $1 \mathrm{~mW}$. 

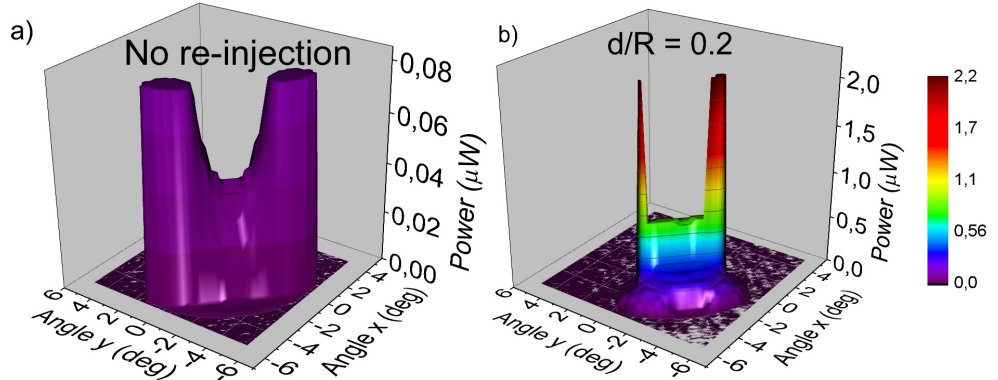

Fig. 2. Angular distribution of cavity transmission power (shown in color) without re-injection mirror (panel a) and with it for $d / R=0.2$ (panel b) for an off-axis radius of $\rho=24 \mathrm{~mm}$.

For $\rho=24 \mathrm{~mm}$ in the case of no re-injection, two symmetrical plateaus for the highest power are formed around $1^{\circ}$ in $\mathrm{x}$ - and $4^{\circ}$ in y-direction and vice versa in Fig. 2(a), where the color indicates the transmission power. For angles around $0^{\circ}$, the transmission power drops as the light travels in a straight line inside the cavity with only a few reflections. The transmission with re-injection mirror for $\mathrm{d} / \mathrm{R}=0.2$ was 28 times higher than without, only achieved in a small range around the same angles as in panel (a). The sets of angles for maximum transmission differed for all $\rho$ and $d / R$, where the most insensitive case was a $\rho=6$ $\mathrm{mm}$ and $\mathrm{d} / \mathrm{R}=0.1$ and the most sensitive for $\rho=24 \mathrm{~mm}$ and $\mathrm{d} / \mathrm{R}=1$.

a)

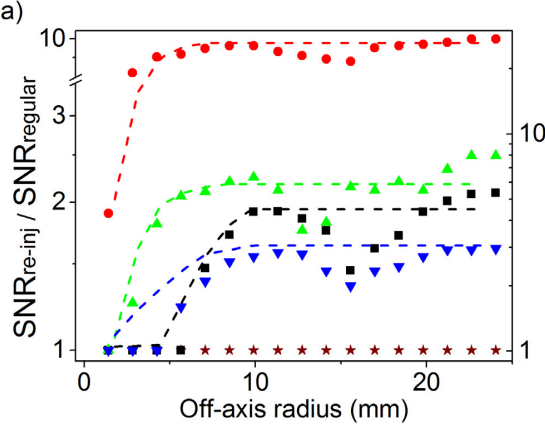

b)

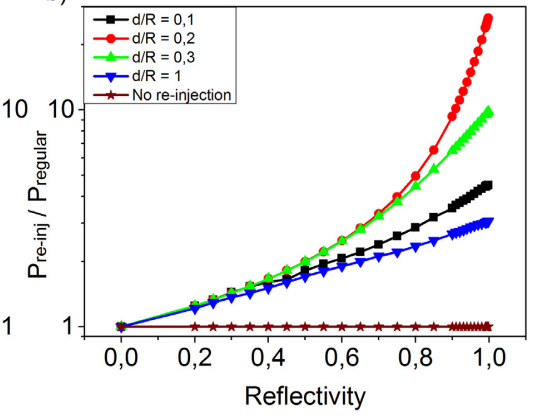

Fig. 3. Simulated and experimental data. Panel a: Ratio of measured SNR (symbols, left axis) and calculated transmission power (dashed lines, right axis) versus off-axis radius at optimum angles for various $\mathrm{d} / \mathrm{R}$ ratios. Panel $\mathrm{b}$ : Simulated power enhancement versus reflectivity of the re-injection mirror for the same $\mathrm{d} / \mathrm{R}$ ratios.

To verify the enhancement effect of the re-injection mirror, the SNR ratio of the ethylene absorption peak has been measured in the absence and presence of the re-injection mirror. The distance $\mathrm{d}$ between the re-injection mirror and the cavity was varied in steps of $5 \mathrm{~cm}$ from 5 $\mathrm{cm}(\mathrm{d} / \mathrm{R}=0.1)$ to $50 \mathrm{~cm}(\mathrm{~d} / \mathrm{R}=1)$ with a fixed $\mathrm{R}=50 \mathrm{~cm}$. At each $\rho$ all three mirrors were optimized according to the simulated data to yield the maximum transmission. The ratio of the measured SNR's in Fig. 3(a) (symbols, left axis) decreased with larger $\mathrm{d} / \mathrm{R}$ and increased with larger radius and agree largely with the corresponding calculated transmission powers (dashed lines, right axis). A ratio of 10 in SNR before and after re-injection was achieved for $d / R=$ 0.2 for the largest radius $\rho=24 \mathrm{~mm}$, which corresponds to a simulated power ratio of 28 . The decrease in SNR in all curves occurred due to the presence of etalons. In Fig. 3(b) simulated data for effect of the reflectivity of the re-injection mirror on the power enhancement is shown for different $\mathrm{d} / \mathrm{R}$ at a fixed radius of $\rho=24 \mathrm{~mm}$. Until a reflectivity of $30 \%$ the power ratio shows no significant difference. At $70 \%$ reflectivity the power ratio amounts to 4 for both $d / R$ $=0.2$ and $\mathrm{d} / \mathrm{R}=0.3$. The highest ratio of 29 was calculated for $\mathrm{d} / \mathrm{R}=0.2$ and $\mathrm{r}=99.8 \%$. 
a)

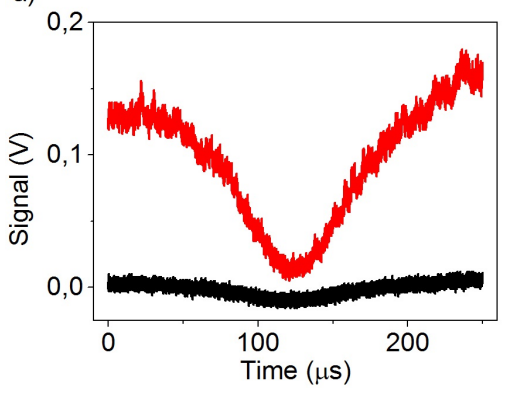

b)

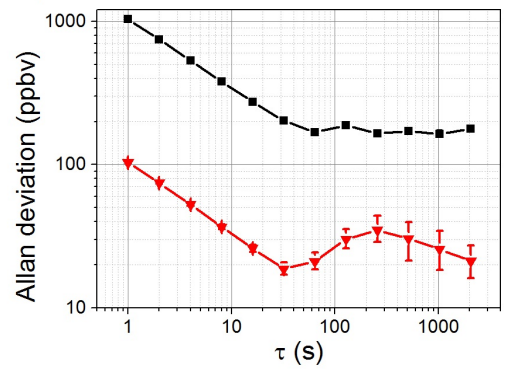

Fig. 4. (a) Absorption spectra of 200 ppbv ethylene at 100 mbar measured with the re-injection mirror (upper curve, red) and without (lower curve, black). (b) Comparison of Allan deviations to determine the detection limit as a function of the integration time. Regular OA-ICOS reached a minimum detection limit of 183 ppv within $128 \mathrm{~s}$ acquisition time (black squares). With the optical enhancement, a detection limit of $18 \mathrm{ppbv}$ in $32 \mathrm{~s}$ was obtained (red triangles).

In order to demonstrate the improved sensitivity using re-injection, we measured the spectrum of $200 \mathrm{ppbv}$ ethylene at 100 mbar with and without re-injection with optimal settings. The result is shown in Fig. 4(a), showing an increase in SNR of one magnitude. We estimated the stability of the system over time by calculating the Allan deviation [16]. In Fig. 4(b) the detection limit of the QCL-based sensor is shown for both the optimum three mirror configuration and to the regular scheme without re-injection. The detection limit for the latter was $1033 \mathrm{ppbv}$ of ethylene for $1 \mathrm{~s}$ averaging ( $3 \sigma$-value) and reached the minimum value of $183 \mathrm{ppbv}$ in $128 \mathrm{~s}$. Using light re-injection technique, a detection limit of $18 \mathrm{ppbv}$ in $32 \mathrm{~s}$ was achieved, which translates to a noise equivalent absorption sensitivity of $1.5 \times 10^{-8} \mathrm{~cm}^{-1} \mathrm{~Hz}^{-}$ $1 / 2$. This represents an enhancement by a factor 10 , in agreement with the improvement in SNR. The number of re-injections can be further increased using differently shaped mirrors (e.g. astigmatic mirrors), before the optical beam exits the hole in the re-injection mirror.

\section{Discussion}

An off-axis system should be optimized before applying the re-injecting technique [17], as the optical enhancement may also increase the optical noise and subsequently reducing the SNR. For example, spurious resonant couplings that produce optical noise increase when beams overlap in the optical cavity, as shown in Fig. 3(a), and the light might be partially lost when focusing on the detector. The noise-related factors have been excluded from the simulation, as the purpose of this work was to provide a fast and reliable prediction tool for the optimum performance of an enhanced spectrometer. These effects can be minimized by changing other parameters of the setup than the simulated ones. In our case, the scanning speed was increased from $4 \mathrm{kHz}$ as in the previous experiment [13] to $7 \mathrm{kHz}$.

The re-injection model presented here can predict the sensitivity enhancement of an existing system with one additional mirror compared to standard OA-ICOS and it can readily be applied to any off-axis configuration. Two routes can be exploited: (1) existing setups can be improved based on their fixed parameters (i.e. laser power, cavity length, mirror reflectivity, etc.) or (2) new systems may be designed for an enhanced scheme depending on the user's requests to gain maximum transmission efficiency. Moreover, using our experimental system as reference, a theoretical prediction of the expected sensitivity and the corresponding optimum conditions can be obtained. Compared to standard OA-ICOS, although the angular dependency was more critical in the enhanced configuration, shown in Fig. 2, the technique preserved the robustness and rather simple alignment, thus ensuring its practicality for field measurements. Potentially, different setups than the one presented here might provide an even higher enhancement. 


\section{Conclusion}

We have demonstrated a versatile simulation tool of an improved three mirror OA-ICOS instrument, which re-injects the light reflected by an optical cavity, and have shown good agreement with experimental results with a QCL at $965 \mathrm{~cm}^{-1}$ for the detection of ethylene. In particular, we optimized the distance between the cavity and the third mirror with respect to the off-axis radius of the cavity and achieved a gain a factor 10 in SNR. Furthermore, our enhanced OA-ICOS system provides real-time measurements of ethylene with a high finesse cavity yielding $18 \mathrm{ppbv}$ in $32 \mathrm{~s}$ averaging time, which is equal to a noise equivalent absorption sensitivity of $\sim 1.5 \times 10^{-8} \mathrm{~cm}^{-1} \mathrm{~Hz}^{-1 / 2}$.

\section{Acknowledgments}

This work was supported by the Foundation for Fundamental Research on Matter under contract N0304M and the European Regional Development Fund, province of Gelderland, GO-EFRO project (no. 2009-010034). The authors would like to thank Jop van Dijk for his contribution to the implementation of the model and Sacco te Lintel Hekkert for fruitful discussions.

\#220903 - \$15.00 USD Received 12 Aug 2014; revised 23 Sep 2014; accepted 30 Sep 2014; published 4 Nov 2014

(C) 2014 OSA 17 November 2014 | Vol. 22, No. 23 | DOI:10.1364/OE.22.027985 | OPTICS EXPRESS 27991 